\title{
The extreme flare in III Zw 2:
}

\section{Evolution of a radio jet in a Seyfert galaxy}

\author{
A. Brunthaler ${ }^{1,2}$, H. Falcke ${ }^{3,4}$, G. C. Bower ${ }^{5}$, M. F. Aller ${ }^{6}$, H. D. Aller ${ }^{6}$, and H. Teräsranta \\ 1 Max-Planck-Institut für Radioastronomie, Auf dem Hügel 69, 53121 Bonn, Germany \\ 2 Joint Institute for VLBI in Europe, Postbus 2, 7990 AA Dwingeloo, The Netherlands \\ e-mail: brunthaler@jive.nl \\ 3 ASTRON, Postbus 2, 7990 AA Dwingeloo, The Netherlands \\ ${ }^{4}$ Department of Astrophysics, Radboud Universiteit Nijmegen, Postbus 9010, 6500 GL Nijmegen, The Netherlands \\ 5 Radio Astronomy Laboratory, University of California at Berkeley, 601 Campbell Hall, CA 94720, USA \\ ${ }^{6}$ University of Michigan, Astronomy Department, Ann Arbor, MI 48109-1090, USA \\ 7 Metsähovi Radio Observatory, Helsinki University of Technology, Metsahovintie 114, 02540 Kylmälä, Finland
}

Received 24 November 2004 / Accepted 24 January 2005

\begin{abstract}
A very detailed monitoring of a radio flare in the Seyfert I galaxy III Zw 2 with the VLA and the VLBA is presented. The relative astrometry in the VLBA observations was precise to a few $\mu$ as. The spectral and spatial evolutions of the source are closely linked, and these observations allowed us to study a textbook example of a synchrotron self-absorbed jet in great detail. We observed a phase where the jet gets frustrated with neither expansion nor spectral evolution. Then the jet breaks free and starts to expand with apparent superluminal motion. This expansion is accompanied by a strong spectral evolution. The results are a good confirmation of synchrotron theory and equipartition for jets.
\end{abstract}

Key words. galaxies: active - galaxies: individual: III Zw 2 - galaxies: jets - galaxies: Seyfert

\section{Introduction}

The radio properties of quasars with otherwise very similar optical properties can be markedly different, as seen in a clear dichotomy between radio-loud and radio-quiet quasars in optically selected samples. Radio-loudness is usually characterized by the radio-to-optical flux ratio. In the PG quasar sample, which is probably the best studied quasar sample in the radio and optical (Kellermann et al. 1989; Boroson \& Green 1992), radio-loud and radio-weak quasars separate cleanly into two distinct populations (e.g. Kellermann et al. 1989).

It is known that radio-loud AGN almost never reside in late type, i.e. spiral galaxies (e.g. Kirhakos et al. 1999; Bahcall et al. 1995), whereas radio-quiet quasars appear both in spiral and in elliptical host galaxies. Furthermore, all relativistically boosted jets with superluminal motion and typical blazars have been detected in early type galaxies (e.g. Scarpa et al. 2000). It is still unclear why AGN in spiral galaxies with the same optical luminosity as their elliptical counterparts, should not be able to produce the powerful relativistic jets seen in radio galaxies.

However, a few sources with intermediate radio-tooptical ratios appear to be neither radio-loud nor radioquiet. They form a distinct subclass with very similar radio morphological and spectral properties. They all have a compact core at arcsecond scales and a flat and variable spectrum in common. These properties are very similar to those of radio cores in radio-loud quasars, but their low radio-tooptical ratio and their low extended steep-spectrum emission is atypical of radio-loud quasars. Miller et al. (1993) and Falcke et al. (1996b) have identified a number of these sources, called "radio-intermediate quasars" (RIQs), and suggested that they might be relativistically boosted radio-weak quasars or "radioweak blazars". This would imply that most, if not all, radioquiet quasars also have relativistic jets. In fact, Very Long Baseline Interferometry (VLBI) observations of radio-quiet quasars already have shown high-brightness temperature radio cores and jets (Falcke et al. 1996a; Blundell \& Beasley 1998). A crucial test of the relativistic jet hypothesis is to search for apparent superluminal motion in these sources, with a prime candidate in the brightest radio source in the RIQ sample, III Zw 2, that we discuss in this paper.

The galaxy III Zw 2 (PG 0007+106, Mrk 1501, $z=0.089$ ) was discovered by Zwicky (1967), then classified as a Seyfert I galaxy (e.g., Arp 1968; Khachikian \& Weedman 1974; Osterbrock 1977), and later also included in the PG quasar sample (Schmidt \& Green 1983). The host galaxy was classified as 
a spiral (e.g. Hutchings \& Campbell 1983) and a disk model was later confirmed by fitting model isophotes to near-IR images (Taylor et al. 1996). A spiral arm was claimed (Hutchings 1983), but recent observations suggest a tidal arm with several knots of star forming regions (Surace et al. 2001). It is the brightest member of a group of galaxies, while an extended low surface brightness emission surrounding all the galaxies suggests that there are perhaps interactions between these galaxies (Surace et al. 2001).

The source has shown extreme variability at radio wavelengths with at least 20-fold increases in radio flux density within 4 years (Aller et al. 1985), and also known to be variable in the optical (Lloyd 1984; Clements et al. 1995) and X-ray (Kaastra \& de Korte 1988). Salvi et al. (2002) compare the long term radio light curves of IIIZw 2 with optical, IR and $\mathrm{X}$-ray light curves and find indications of correlated flux variations from radio to $\mathrm{X}$-ray wavelengths. Unfortunately, the time sampling at other than radio wavelengths is very poor.

The radio source III Zw 2 is a core-dominated flat-spectrum AGN with only a faint extended structure (see Unger et al. 1987; and Sect. 3.1). The weak extended radio emission and the host galaxy are quite typical of a Seyfert galaxy. Its [OIII] luminosity is a mere factor three brighter than that of a bright Seyfert galaxy like Mrk 3 (e.g. Alonso-Herrero et al. 1997), which explains why it has been classified as either a Seyfert galaxy or a quasar. In this luminosity region a distinction between the two may not be very significant.

Earlier VLBI observations of the source have only shown an unresolved high-brightness temperature core (Falcke et al. 1996b; Kellermann et al. 1998), and Millimeter-VLBI observations by Falcke et al. (1999) just barely resolved the source into two very compact components. A broadband radio spectrum showed a highly peaked spectrum that was well explained by a very compact source and synchrotron self-absorption.

The unique and simple structure and timescales of radio outbursts within 5 years makes IIIZw 2 an ideal source for studying radio-jet evolution also relevant to radio galaxies. In Sect. 2, we will describe our Very Large Array (VLA) and Very Long Baseline Array (VLBA) observations, before results from VLA monitoring are presented in Sects. 3.1-3.3. We then describe the results from VLBI observations in Sect. 3.4. In Sect. 4 we will discuss the results.

\section{Observations}

Since III Zw 2 started a new major radio outburst 1996 we initiated a Target-of-Opportunity program to monitor the spectral evolution of this burst with the VLA and its structural evolution with the VLBA and its excellent relative astrometry of the component separation.

We observed IIIZw 2 with the VLA 41 times from September 1998 until September 2001 at intervals of roughly one month. The observations were made at six frequencies ranging from $1.4 \mathrm{GHz}$ to $43 \mathrm{GHz}$. Results from an observation on 21 May 1998 at $350 \mathrm{MHz}$ and on 7 July 1999 at $327.5 \mathrm{MHz}$ are also presented here. The source $3 \mathrm{C} 48$ was used as the primary flux density calibrator, and III Zw 2 was self-calibrated
Table 1. Total observing time $t_{\mathrm{obs}}$, fraction of observing time of III Zw 2 at $43 \mathrm{GHz} f_{43}$ and $15 \mathrm{GHz} f_{15}$, and fraction of observing time of phase-reference quasar $f_{\text {quasar }}$ for the VLBA observations.

\begin{tabular}{lllcl}
\hline \hline Date & $t_{\text {obs }}$ & $f_{43}$ & $f_{15}$ & $f_{\text {quasar }}$ \\
\hline $1998 / 02 / 16$ & $8 \mathrm{~h}$ & 0.75 & 0.25 & - \\
$1998 / 06 / 13$ & $9 \mathrm{~h}$ & 0.75 & 0.25 & - \\
$1998 / 09 / 14$ & $8 \mathrm{~h}$ & 0.75 & 0.25 & - \\
$1998 / 12 / 12$ & $8 \mathrm{~h}$ & 0.75 & 0.25 & - \\
$1999 / 07 / 15$ & $6 \mathrm{~h}$ & 0.75 & 0.25 & - \\
$1999 / 11 / 12$ & $8 \mathrm{~h}$ & 0.33 & 0.33 & 0.33 \\
$2000 / 07 / 22$ & $6 \mathrm{~h}$ & 0.33 & 0.33 & 0.33 \\
$2000 / 08 / 27$ & $8 \mathrm{~h}$ & 0.33 & 0.33 & 0.33 \\
$2000 / 09 / 06$ & $8 \mathrm{~h}$ & 0.33 & 0.33 & 0.33 \\
\hline
\end{tabular}

and mapped with the Astronomical Image Processing System (AIPS).

We also used the monitoring data at 8 and $15 \mathrm{GHz}$ obtained with the Michigan $26 \mathrm{~m}$ telescope, and at 22 and $37 \mathrm{GHz}$ with the Metsähovi radio telescope. The single dish data are important for placing the VLA and VLBA data in context, as they offer a larger time window and are more closely spaced.

We observed IIIZw 2 with the VLBA nine times over a period of 2.5 years at 15 and $43 \mathrm{GHz}$. Details of this observations are given in Table 1. In the last four epochs we included the background quasar $\mathrm{J} 0011+0823$ at $15 \mathrm{GHz}$ as phase-reference source. For the second epoch, we used the Effelsberg $100 \mathrm{~m}$ telescope in combination with the VLBA. We observed in all epochs four $8 \mathrm{MHz}$ bands, each at right and left circular polarization. The initial calibration was performed with the AIPS package. A-priori amplitude calibration was applied using system temperature measurements and standard gain curves. Fringes were found in the III Zw 2 data on all baselines. The data were self-calibrated and mapped using the software package DIFMAP (Shepherd et al. 1994). We started with phase-only self-calibration and later included phase-amplitude self-calibration with solution intervals slowly decreasing down to one minute. Results of the first five VLBA observations at $43 \mathrm{GHz}$ were reported by Brunthaler et al. (2000).

\section{Results}

\subsection{Extended emission of III Zw2}

Unger et al. (1987) discovered a weak radio component 15.4" southwest of the nucleus. This corresponds to a size of $23 \mathrm{kpc}$ at an angular size distance of $d_{\mathrm{A}} \sim 307.4 \mathrm{Mpc}\left(H_{0}=\right.$ $75 \mathrm{~km} \mathrm{~s}^{-1} \mathrm{Mpc}^{-1}, q_{0}=0.5$ as used in this paper). This detection was confirmed later (Kukula et al. 1998; Falcke et al. 1999), but no additional extended radio emission was found.

To study the extended structure in more detail, we combined the raw data of eleven VLA observations, using data from the VLA in A, B, C and D configuration. Since the nucleus is highly variable, we subtracted it from the uv-data before combining the data. The combined data set was then self-calibrated and mapped, and the combined VLA maps at 1.4, 4.8, 8.4, and $15 \mathrm{GHz}$ are shown in Figs. 1 and 2. The 4.8, 8.4, and $15 \mathrm{GHz}$ maps were convolved with a large beam of $2 \times 2$ arcsec 


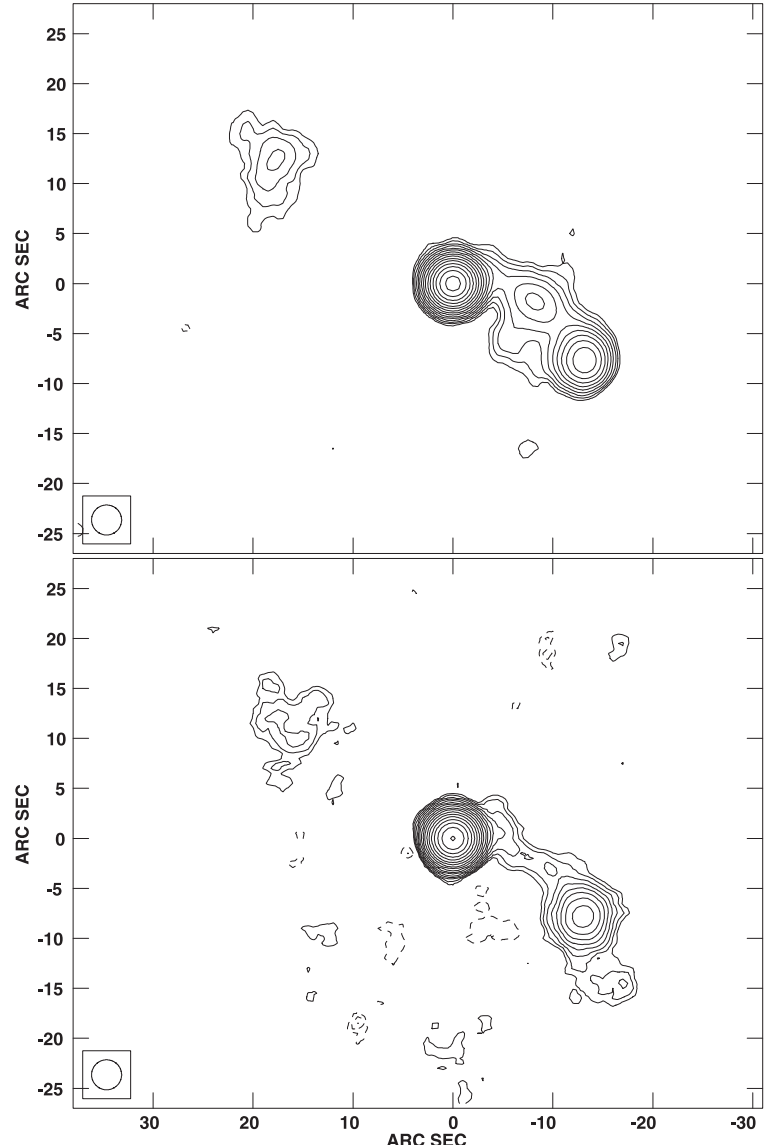

Fig. 1. Combined VLA map of 11 epochs (A, B, C and D-array) of III Zw 2 at $1.4 \mathrm{GHz}$ (top) and at $4.8 \mathrm{GHz}$ (bottom). All maps were convolved with a beam of $2 \times 2$ arcsec to detect faint extended emission. The contours start at $0.26 \mathrm{mJy}$ at $1.4 \mathrm{GHz}$ and $0.15 \mathrm{mJy}$ at $4.8 \mathrm{GHz}$, and increase with a factor of $\sqrt{2}$.

to detect faint extended structure. We detected the southwestern component at all four frequencies. This radio lobe or hotspot is connected to the nucleus with a jet-like structure visible at 1.4, 4.8, and $8.4 \mathrm{GHz}$. At $1.4 \mathrm{GHz}$ one sees an indication that the jet is ejected in a northwestern direction and gets deflected by almost $90^{\circ}$ towards the southwestern lobe. This is also in accordance with the direction of the jet on sub-parsec scales (see Sect. 3.4).

We also discovered a weaker secondary radio lobe $21.9^{\prime \prime}$ $(32.6 \mathrm{kpc})$ on the opposite side of the galaxy at 1.4 to $8.4 \mathrm{GHz}$. If one assumes equal expansion velocities for both lobes, a simple time travel argument (e.g., Ryle \& Longair 1967) would suggest that the weaker northeastern lobe is approaching and the brighter southwestern lobe is receding. However, this scenario cannot explain the differences in flux density between the two lobes. One would expect the approaching lobe to be brighter, not fainter, due to relativistic boosting of the emission. Hence it is more likely that the armlength difference is explained by an asymmetric expansion of the the two lobes due to different intrinsic velocities or differences in the ambient medium; i.e. the medium in the southwest of III Zw 2 has a higher density than the medium in the northeast. This

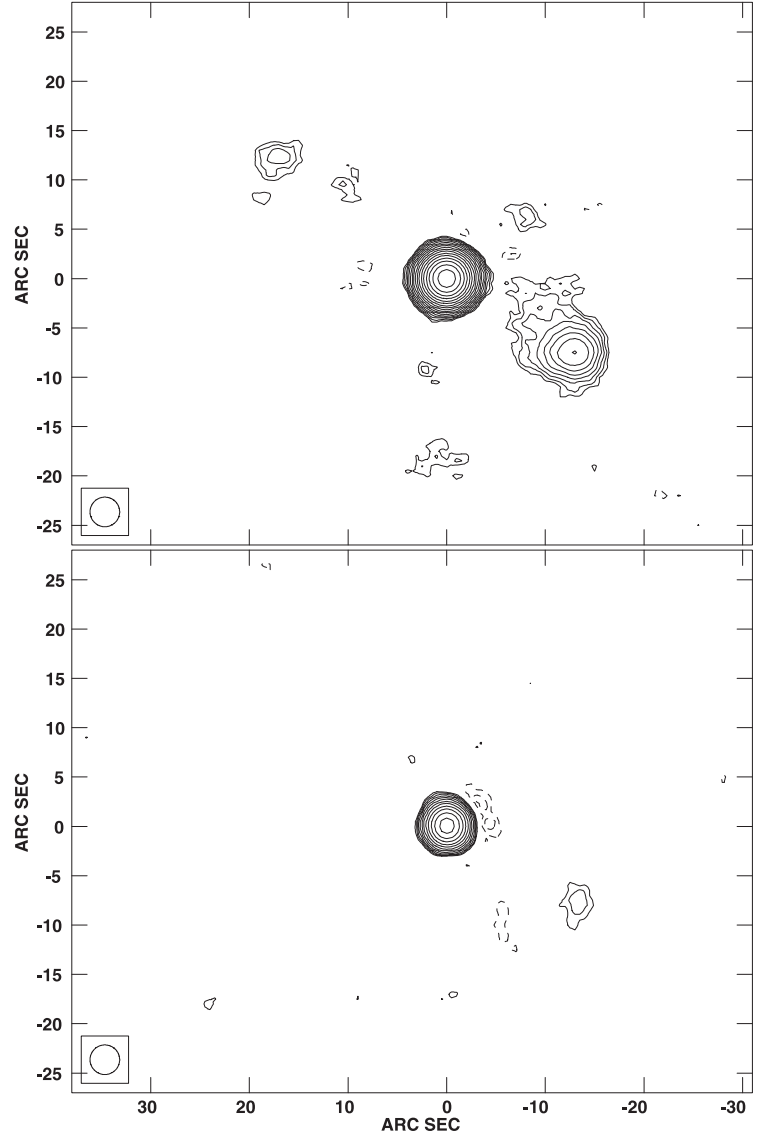

Fig. 2. Combined VLA map of 11 epochs (A, B, C and D-array) of III Zw 2 at $8.4 \mathrm{GHz}$ (top) and $15 \mathrm{GHz}$ (bottom). All maps were convolved with a beam of $2 \times 2$ arcsec to detect faint extended emission. The contours start at $0.12 \mathrm{mJy}$ at $8.4 \mathrm{GHz}$ and $0.6 \mathrm{mJy}$ at $15 \mathrm{GHz}$, and increase with a factor of $\sqrt{2}$.

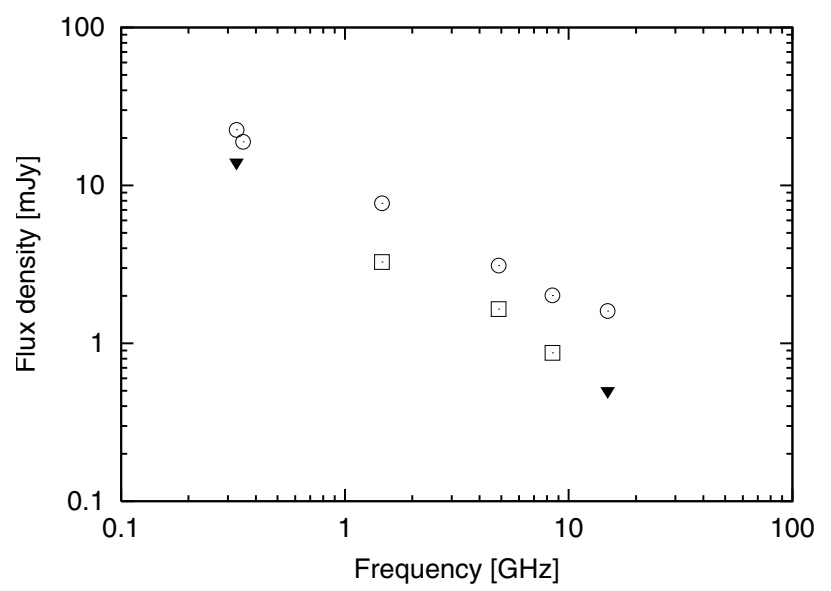

Fig. 3. Spectra of the southwestern (circles) and northeastern (squares) radio lobes. The black triangles are upper limits for the northeastern lobe.

is supported by the fact that there is a close companion galaxy only $\sim 30^{\prime \prime}$ to the south.

The spectra of the two radio lobes are shown in Fig. 3. The 350 and $327.5 \mathrm{MHz}$ data were from our observations on 21 May 1998 and 7 July 1999, respectively, where we also detected the southwestern component. Both radio lobes have a 
steep spectrum with spectral indices of $\alpha=-0.57$ to -1.15 . Values around -0.7 are typical for synchrotron emission of optically thin radio lobes of radio galaxies. There is no break or steepening of the spectrum towards higher frequencies, which indicates that both radio lobes are still active and powered by the central engine. Otherwise, the high energy electrons would have lost most of their energy due to radiation losses, thereby leading to a steepening in the spectrum at higher frequencies.

\subsection{Variability}

The core of III Zw 2 shows extreme variability at radio wavelengths. Long term radio light curves of this source spanning more than 20 years are shown in Fig. 4 using data from Michigan, Metsähovi, and the VLA. One can see major flares with 30-fold increases in radio flux density within two years. These major flares occur roughly every five years with subflares on shorter timescales.

The outburst discussed in this paper started in 1996 and we monitored this flare regularly with the VLA; good time sampling of one observation each month allowed us to study this outburst in great detail. Lightcurves from the most recent flare at six frequencies from 1.4 to $43 \mathrm{GHz}$ are shown in Figs. 5 and 6 together with our best model fits to the data. Since the Michigan data at $8 \mathrm{GHz}$ is rather noisy compared to the other frequencies and the VLA data, we used only our VLA monitoring data for the fits at this frequency. At 15, 22, and $37 \mathrm{GHz}$ we used the VLA data, as well as the Michigan and Metsähovi data.

First we fitted a linear rise and decay to the flare. The rise is consistent with a linear fit at all frequencies. The decay is also linear at $4.8 \mathrm{GHz}$. At higher frequencies, the decay is linear only for a short time period and deviates significantly from a linear behavior at later times. Thus we used only the linear part of the lightcurves for our fits.

Decay at higher frequencies can be fitted much better with an exponential decay; thus we fitted also an exponential rise and decay,

$$
\begin{aligned}
& S(t)=S_{0} \mathrm{e}^{\left(t_{0}-t\right) / \tau_{\mathrm{r}}}, \\
& S(t)=S_{0} \mathrm{e}^{\left(t_{0}-t\right) / \tau_{\mathrm{d}}},
\end{aligned}
$$

to the lightcurves. The fitting parameters are (i) $S_{0}$, the maximum amplitude of the flare, and (ii) the flare rise and decay timescales $\tau_{\mathrm{r}}$ and $\tau_{\mathrm{d}}$. The epochs of the flare maximum $t_{0}$ were taken from the observation with the highest flux density. Increase in the lightcurves can also be fitted by an exponential rise, and the exponential decay fits the outburst until a new smaller flare starts. The slopes of the linear rise $\left(a_{\mathrm{r}}\right)$ and decay $\left(a_{\mathrm{d}}\right)$, the exponential rise and decay timescales $\tau_{\mathrm{r}}$ and $\tau_{\mathrm{d}}$, and the epoch of the flare maximum $t_{0}$ are listed in Table 2 for all six frequencies.

The lightcurve reaches its peak first at higher frequencies. The time-lag between the peak at $43 \mathrm{GHz}$ and the peak at lower frequencies is shown in Fig. 7 and is best fit with a $\delta t \propto v^{-1.1}$ power law. The rise and decay are faster at higher frequencies than at lower frequencies, which is typical behavior for flares in AGN (e.g. Türler et al. 1999).

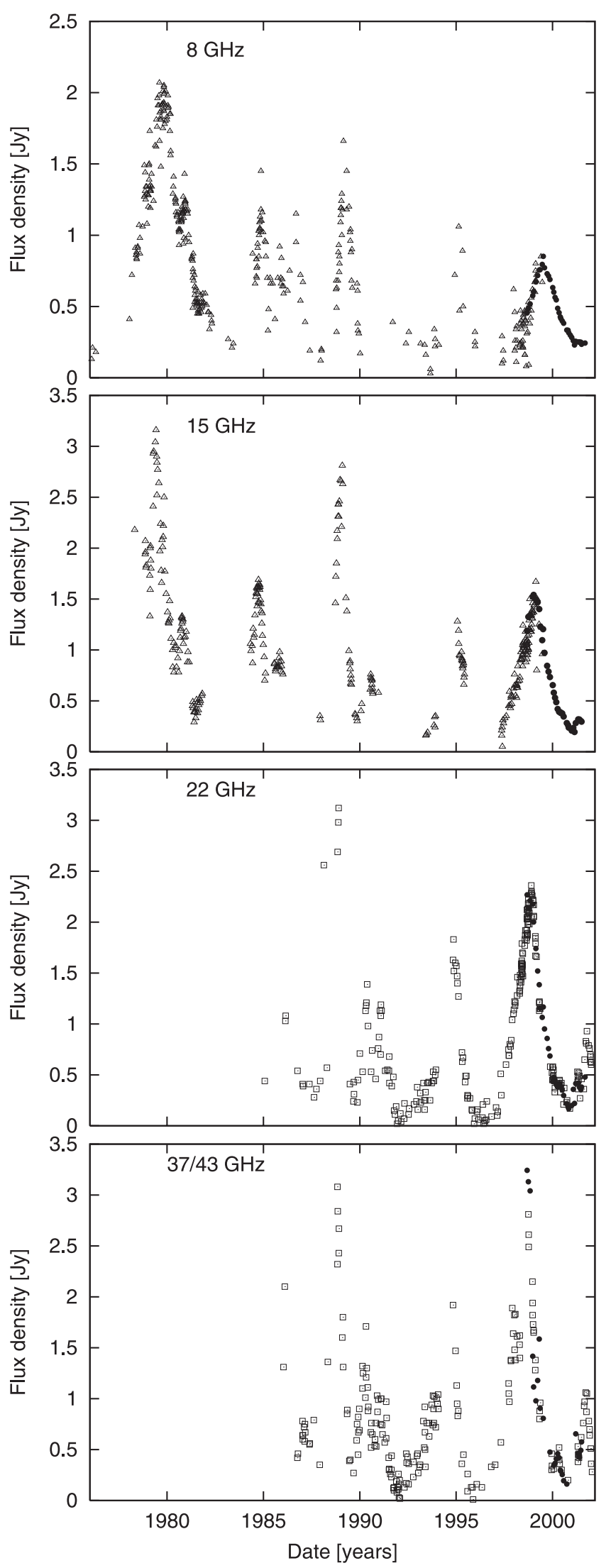

Fig. 4. Radio light curves of III Zw 2. The triangles at 8 and $15 \mathrm{GHz}$ are from the Michigan monitoring program, the squares at 22 and $37 \mathrm{GHz}$ are from the Metsähovi monitoring program. The circles are our VLA observations at 8, 15, 22, and $43 \mathrm{GHz}$. 


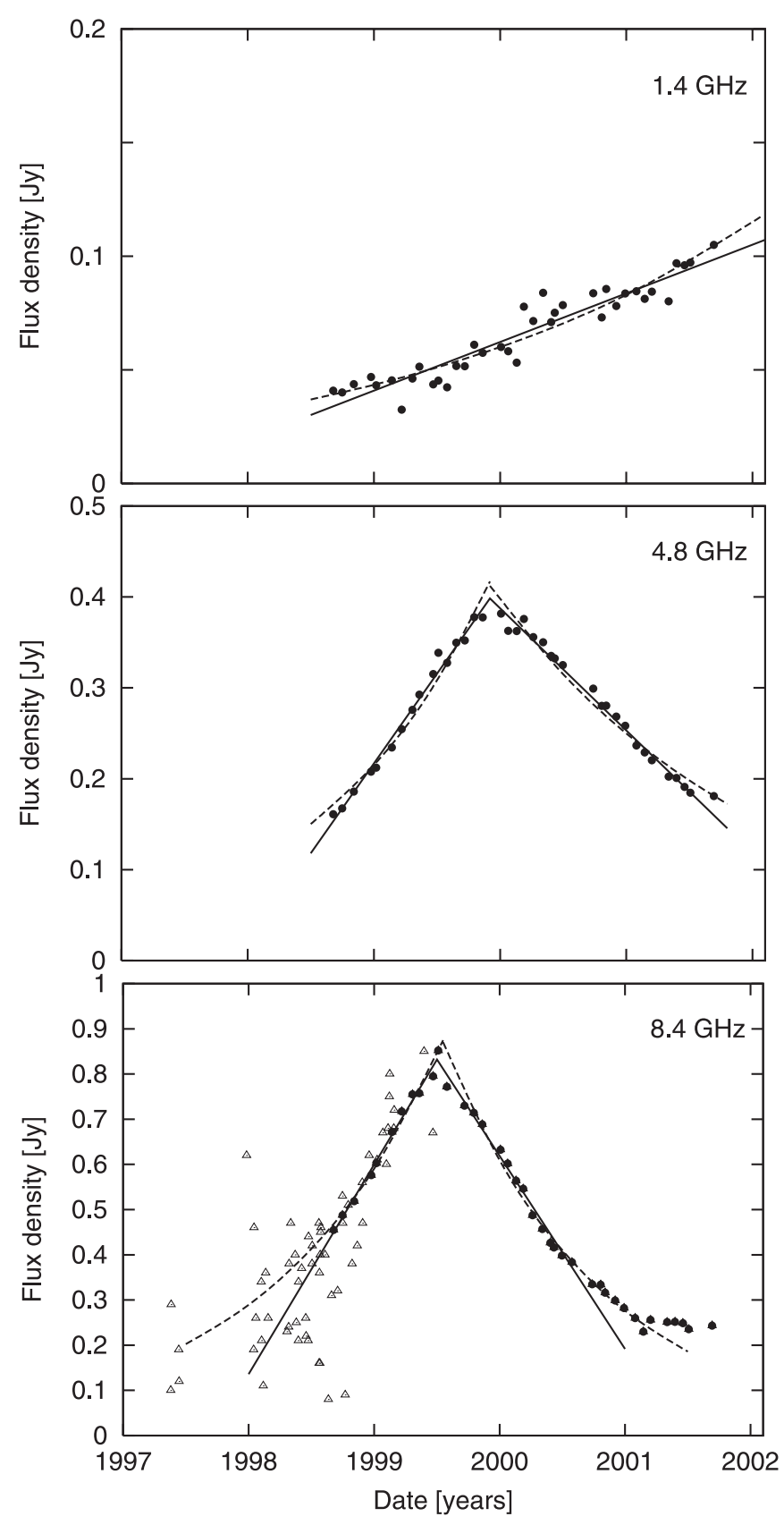

Fig. 5. Radio light curves of the recent flare in III Zw 2 at 1.4, 4.8 and 8.4 GHz. Circles are our VLA observations, and triangles are from the Michigan monitoring program. Solid lines are our linear rise and decline fits, while dashed lines are the fitted exponential rise and decay.

At $4.8 \mathrm{GHz}$ the rise is faster than the decay, while at 15,22 , and $43 \mathrm{GHz}$ the rise is slower than the decay. This is the case for both the linear and exponential fits to the flare. At $8.4 \mathrm{GHz}$ the linear rise is slightly slower than the linear decay, while the exponential rise is slightly faster than the exponential decay. The rise and decay timescales are plotted as a function of frequency in Fig. 8. The data can be fitted with power laws $\tau_{\mathrm{r}} \propto v^{-0.2}$ and $\tau_{\mathrm{d}} \propto v^{-0.7}$, except for the rise timescale at $1.4 \mathrm{GHz}$ which deviates from it. However, the quiescence flux at $1.4 \mathrm{GHz}$ is comparable to the flux density of the outburst and will affect this data point.
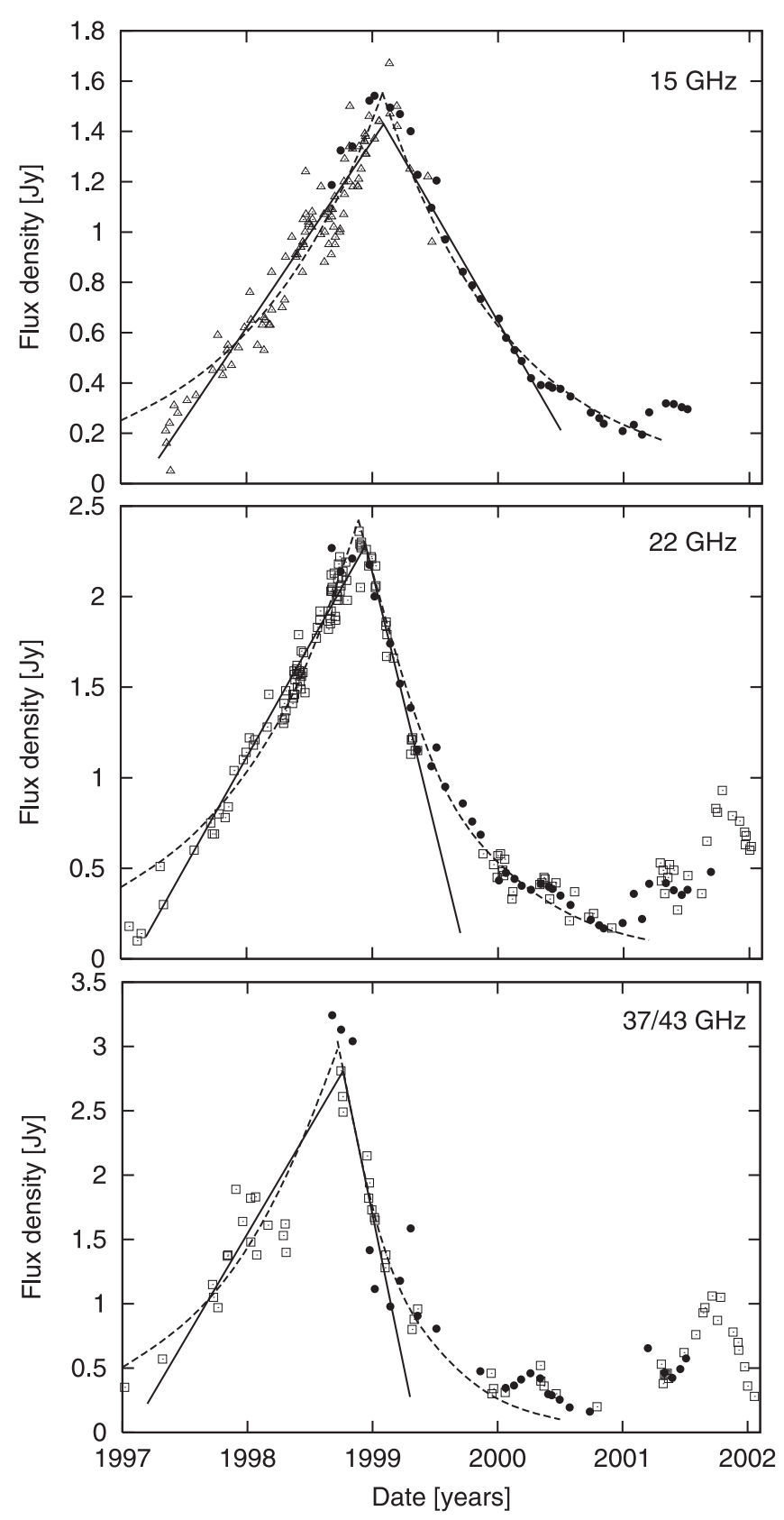

Fig. 6. Radio light curves of the recent flare in III Zw 2 at 15,22 and 37/43 GHz. Circles are our VLA observations, and triangles are from the Michigan monitoring program, and squares are from the Metsähovi monitoring program. Solid lines are our linear rise and decline fits, while dashed lines are the fitted exponential rise and decay.

The low dependence of the rise timescale on frequency indicates that optical depth effects are not very important during the rise, in contrast to the decay where optical depth effects are clearly important.

Valtaoja et al. (1999) modeled the radio lightcurves of flares at 22 and $37 \mathrm{GHz}$ in 85 extragalactic radio sources. They fitted an exponential curve to the rise and decay and found that in virtually all flares a good model-fit could be obtained using a constant ratio between decay and rise timescale of $\tau_{\mathrm{d}}=1.3 \tau_{\mathrm{r}}$; i.e. the rise is faster than the decay. These flares can be identified with the ejection of new VLBI components in the jets. 
Table 2. Fitting information: the slopes of the linear rise $\left(a_{\mathrm{r}}\right)$ and decay $\left(a_{\mathrm{d}}\right)$, the exponential rise and decay timescales $\tau_{\mathrm{r}}$ and $\tau_{\mathrm{d}}$, and the epoch of the flare maximum $t_{0}$.

\begin{tabular}{|c|c|c|c|c|c|}
\hline $\begin{array}{c}\text { Frequency } \\
{[\mathrm{GHz}]}\end{array}$ & $\begin{array}{l}a_{\mathrm{r}} \\
{[\mathrm{Jy}}\end{array}$ & $\begin{array}{r}a_{\mathrm{d}} \\
\left.\mathrm{r}^{-1}\right]\end{array}$ & $\begin{array}{l}\tau_{\mathrm{r}} \\
{[\mathrm{yr}}\end{array}$ & $\tau_{\mathrm{d}}$ & $\begin{array}{c}t_{0} \\
{[\mathrm{yr}]}\end{array}$ \\
\hline 1.4 & 0.021 & - & -3.088 & - & - \\
\hline 4.8 & 0.20 & -0.14 & -1.39 & 2.16 & 2000.1 \\
\hline 8.4 & 0.46 & -0.43 & -1.40 & 1.26 & 1999.5 \\
\hline 15 & 0.74 & -0.86 & -1.14 & 1.01 & 1999.1 \\
\hline 22 & 1.24 & -2.85 & -1.04 & 0.73 & 1998.9 \\
\hline 43 & 1.65 & -4.73 & -0.96 & 0.53 & 1998.7 \\
\hline
\end{tabular}

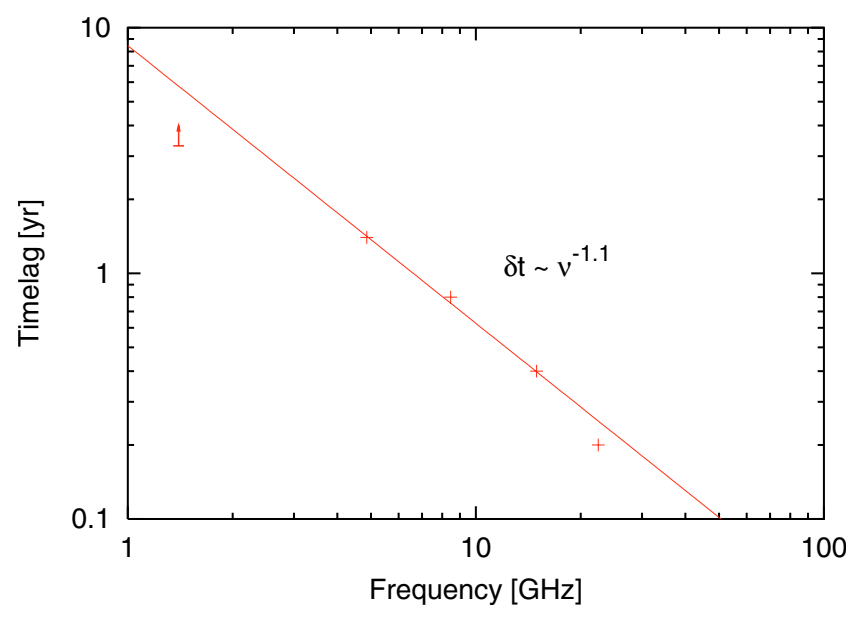

Fig. 7. Time-lag between the peak at $43 \mathrm{GHz}$ and the peak at the other frequencies. The data point at $1.4 \mathrm{GHz}$ is a lower limit.

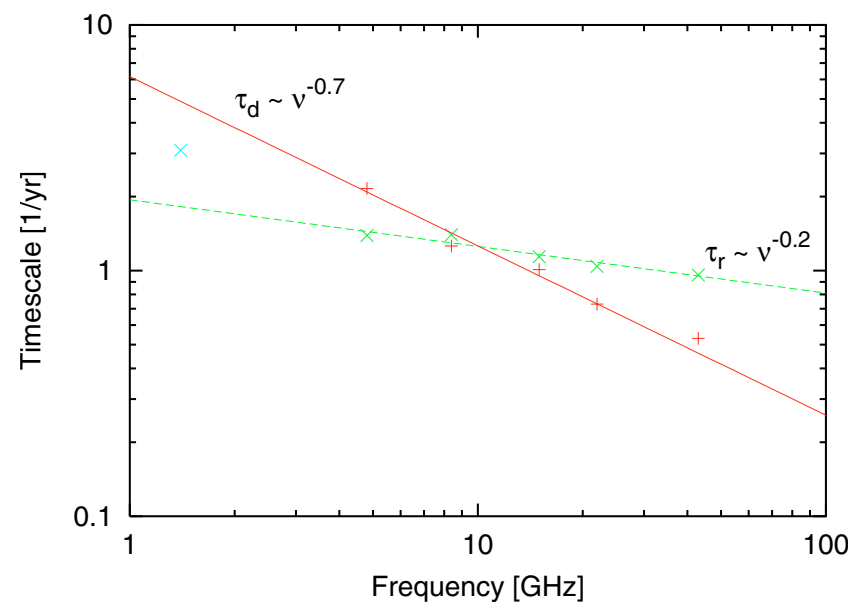

Fig. 8. Rise $(\times)$ and decay $(+)$ timescales for all frequencies.

The fact that the lightcurves of IIIZw 2 at 15, 22, and $43 \mathrm{GHz}$ show the opposite behavior with ratios of $\tau_{\mathrm{d}} / \tau_{\mathrm{r}} \approx$ $0.6-0.9$ - i.e. the decay is faster than the rise - indicates that different physical processes are involved in this source. However, that the flare is composed of two closely spaced flares cannot be excluded.

\subsection{Spectral evolution}

During the outburst the spectrum of the source also showed variations. An almost simultaneous broadband radio spectrum from 1.4 to $660 \mathrm{GHz}$ during the increase in flux density in May 1998 was presented in Falcke et al. (1999). The spectrum was highly inverted at centimeter wavelengths $(\alpha=+1.9 \pm 0.1)$ with a turnover frequency around $43 \mathrm{GHz}$. At frequencies above $43 \mathrm{GHz}$ the spectrum became steep with a spectral index of $\alpha=-0.75 \pm 0.15$; i.e. a textbook-like synchrotron spectrum.

Our VLA monitoring of the spectral evolution started in September 1998. Four of the 41 epochs yielded either no or bad data due to either bad weather or hardware failure. We fitted the remaining spectra with a broken power-law plus a flat and constant quiescence spectrum $S_{\mathrm{q}}$,

$S(v)=S_{0}\left(\frac{v}{v_{0}}\right)^{k}\left(1-\mathrm{e}^{-\left(\frac{v}{v_{0}}\right)^{l-k}}\right)+S_{\mathrm{q}}$,

where $k$ and $l$ are the spectral indices of the rising and declining parts of the spectrum. $S_{0}$ and $v_{0}$ are fitting parameters and are not exactly equal to the maximum flux density and the peak frequency of the fitted spectrum. We assume a flat spectrum for the quiescence flux which is typical for quiescent cores in active galactic nuclei. Since we could fit all epochs with a value of $25 \mathrm{mJy}$, we adopted this value for all epochs.

The approach with a broken power-law has the disadvantage of causing ambiguities in the parameters, if the peak of the spectrum falls outside the frequencies covered in the observations, which was the case in our first three observations in September 1998, October 1998, and November 1998. The spectral shape in these three epochs was very similar to the May 1998 spectrum: highly inverted at centimeter wavelengths, a flattening towards higher frequencies, and possibly a turnover around $43 \mathrm{GHz}$, but with higher flux densities (see Fig. 9, top). Thus we assumed the spectral index of the declining part of the spectrum to be $l=-0.75$, the value from the May 1998 observation. This is a reasonable assumption since the overall spectral shape did not change significantly between May 1998 and September 1998.

The temporal evolution of the fitting parameters $v_{0}, k$, and $l$ can be seen in Figs. 10 and 11. In some epochs we covered only 5 frequencies from 1.4 to $22 \mathrm{GHz}$. The absence of the $43 \mathrm{GHz}$ flux density in these epochs could bias the results of the spectral fitting. Thus we marked the epochs with only 5 frequencies in Fig. 10 with triangles while the epochs with 6 frequencies are indicated by circles. One can see that the fits of both subsets are in good agreement.

After November 1998 the spectrum underwent a dramatic change (see also Fig. 9). The turnover frequency $v_{0}$, which stayed roughly constant at around $30 \mathrm{GHz}$ from May 1998 until November 1998, dropped to $23 \mathrm{GHz}$ in December 1998. In the following months, the turnover dropped further until it reached $10 \mathrm{GHz}$ in June 1999. During the next year the turnover frequency showed only smaller and slower variations and stayed roughly constant at $\sim 7 \mathrm{GHz}$. The drop in turnover frequency to $4 \mathrm{GHz}$ in the first months of 2000 can be explained by the onset of a new minor flare at high frequencies (see the $43 \mathrm{GHz}$ lightcurve in Fig. 6). The new flare caused a flattening of the 


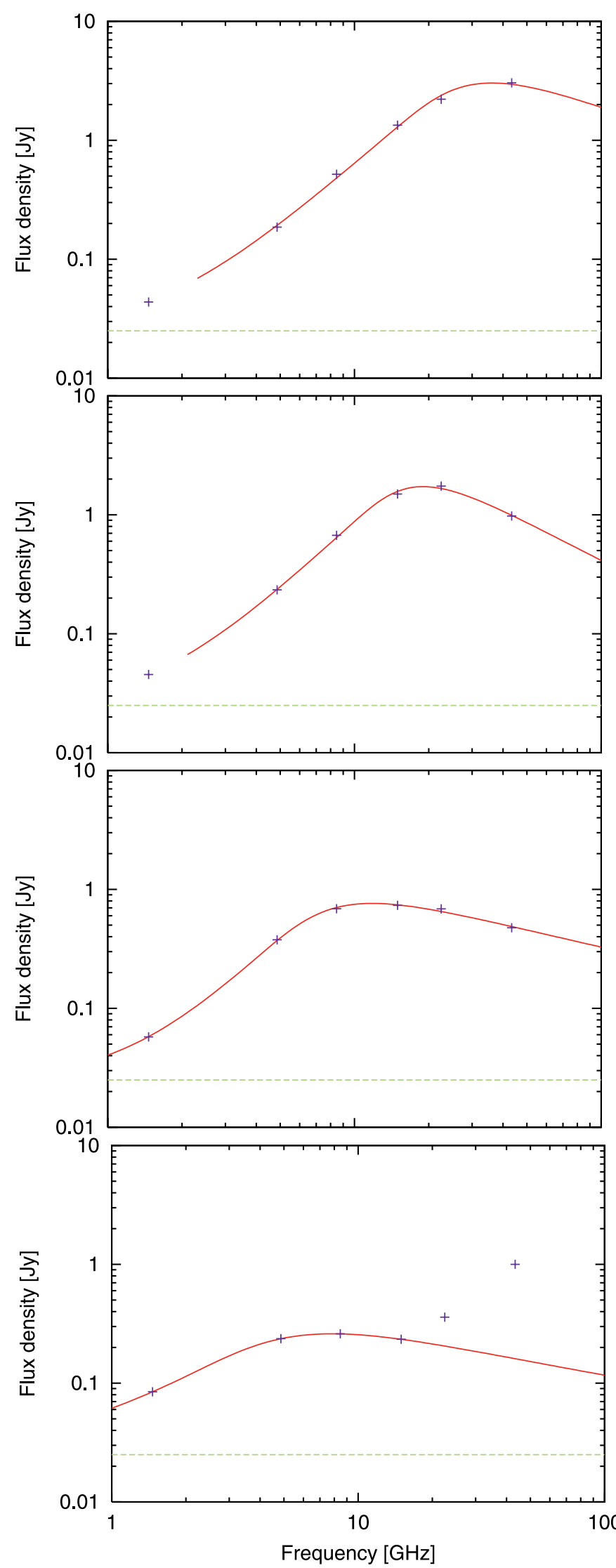

Fig. 9. Spectra of III Zw 2 on 4 November 1998 (top), 22 February 1999 (middle), 12 November 1999 (middle), and 30 January 2001 (bottom) together with the quiescent spectrum (horizontal line) and the best fit spectrum.

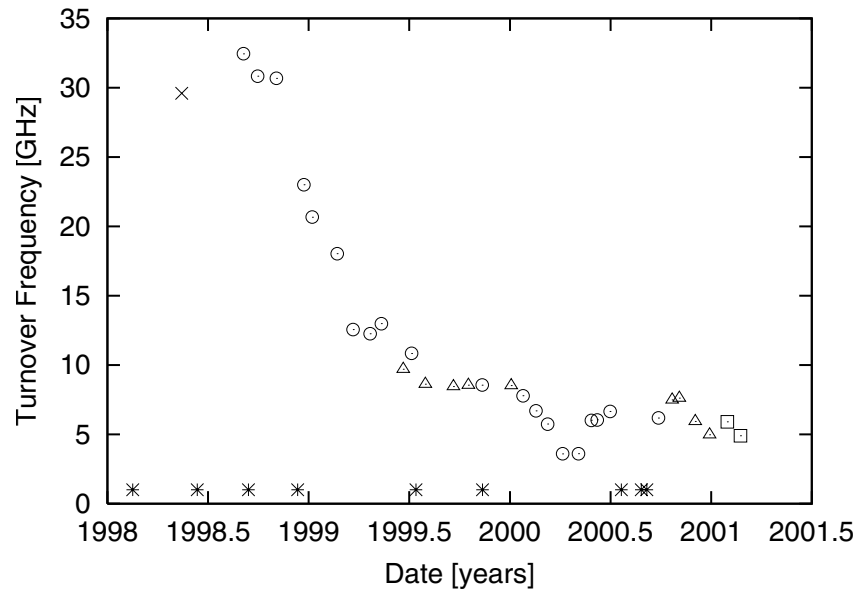

Fig. 10. Evolution of the "turnover frequency" $v_{0}$. Circles mark epochs with 6 observed frequencies $(1.4-43 \mathrm{GHz})$ and triangles epochs with 5 observed frequencies $(1.4-22 \mathrm{GHz})$. In the last two epochs only 4 frequencies $(1.4-15 \mathrm{GHz})$ were used because of a new outburst at high frequencies. The cross is the turnover frequency of the spectrum in May 1998, where the $1-43 \mathrm{GHz}$ data is taken from Falcke et al. (1999). The asterisks mark the epochs of our VLBA observations.

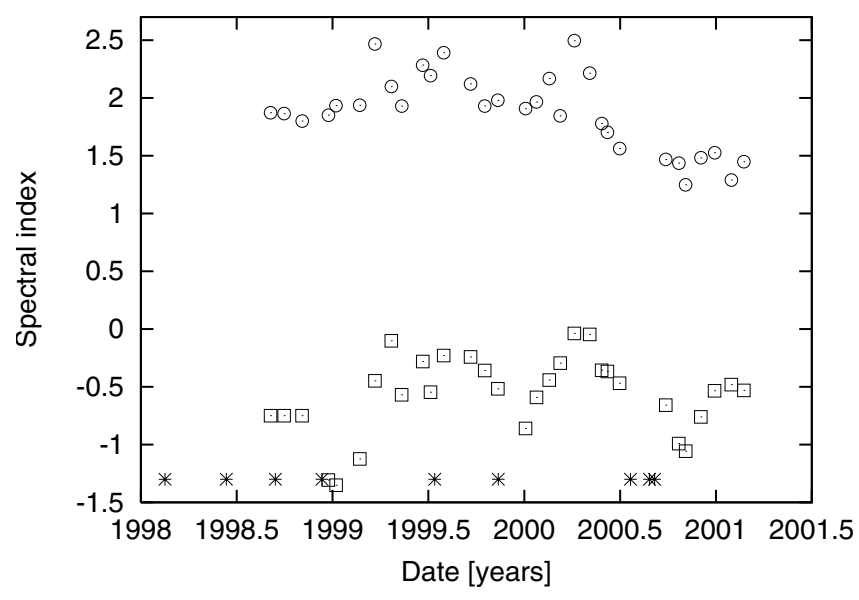

Fig. 11. Evolution of the spectral indices $k$ (circles) and $l$ (squares). The asterisks mark the epochs of our VLBA observations.

optical thin part of the spectrum and a shift of the turnover to lower frequencies. The flattening can also be seen in Fig. 11 where the spectral index $l$ changes from $\sim-0.9$ to $\sim-0.1$ during that time. The spectral index in the optical thick part of the spectrum $k$ stays at $\sim 2$ and slowly flattens towards later times.

A new strong outburst started at high frequencies in January 2001 (see 22 and $43 \mathrm{GHz}$ lightcurves in Fig. 6) so one would have to model two independent broken power-laws to the spectrum. Since one broken power-law is characterized by four parameters $S_{0}, v_{0}, k$, and $l$, our six data points in each spectrum are not sufficient to model two independent components with four parameters each. In the first two epochs of the new flare, only the 22 and $43 \mathrm{GHz}$ data were affected so we fitted the broken power-law to the remaining four frequencies.

The fast change in peak frequency also implies a strong morphological change, i.e. a rapid expansion. This prediction was tested by VLBI observations described in the next section. 
Table 3. Flux densities $S$, separation $D$, and position angle PA of the two outermost point-like components of our model-fits to the $43 \mathrm{GHz}$ uv-data.

\begin{tabular}{llllcl}
\hline \hline Date & $S_{1}[\mathrm{Jy}]$ & $S_{2}[\mathrm{Jy}]$ & $S_{3}[\mathrm{Jy}]$ & $D[\mathrm{mas}]$ & $\mathrm{PA}$ \\
\hline $1998 / 02 / 16$ & 0.93 & 0.58 & - & 0.075 & $-84^{\circ}$ \\
$1998 / 06 / 13$ & 1.03 & 0.65 & - & 0.077 & $-78^{\circ}$ \\
$1998 / 09 / 14$ & 1.60 & 1.27 & - & 0.077 & $-72^{\circ}$ \\
$1998 / 12 / 12$ & 0.86 & 0.86 & - & 0.106 & $-63^{\circ}$ \\
$1999 / 07 / 15$ & 0.56 & 0.26 & 0.08 & 0.245 & $-71^{\circ}$ \\
$1999 / 11 / 12$ & 0.08 & 0.23 & 0.05 & 0.246 & $-73^{\circ}$ \\
\hline
\end{tabular}

\subsection{Structural evolution}

\subsection{1. $43 \mathrm{GHz}$ results}

The first three VLBA observations were made during the first phase of the flare, noticeable by the increase in flux density and a roughly constant spectral peak above $30 \mathrm{GHz}$. The constancy of the peak frequency indicates no structural change, since the turnover is caused by synchrotron self-absorption (Falcke et al. 1999). The source is slightly resolved, and the long baselines show non-zero closure phases, indicating an asymmetric structure. Two point-like components were fitted to the uv-data to represent the extent of the source, which shows no structural change (see Fig. 12); and the separation of the two components during this phase of the flare stayed constant at $\sim 76 \mu \mathrm{as}$, corresponding to $\sim 0.11 \mathrm{pc}$. The excellent agreement within $2 \mu \mathrm{as}$ between the first three epochs shows the high quality of the data and the accuracy of the relative astrometry.

After November 1998, the VLA monitoring showed a dramatic change in the spectrum. The peak frequency dropped quickly to $10 \mathrm{GHz}$ within a few months (Fig. 10). In the framework of a simple equipartition jet model with $R \propto v_{\text {ssa }}^{-1}$ dependence (e.g., Blandford \& Konigl 1979; Falcke \& Biermann 1995 ) one would expect rapid expansion. With a source size of $0.11 \mathrm{pc}$ and a turnover frequency of $33 \mathrm{GHz}$ in the first phase with no expansion, one expects a source size of $0.36 \mathrm{pc}$ for a self-absorption frequency of $10 \mathrm{GHz}$ according to the spectral evolution.

Indeed, the fourth VLBA epoch, observed only one month after the start of the spectral evolution, shows initial signs of an expansion. The fifth epoch shows a dramatic structural change (see Fig. 12), to the point where a model of three point-like components is required to describe the data now. The separation between the outer components is now $\sim 245 \mu$ as corresponding to $\sim 0.37 \mathrm{pc}$. This is in good agreement with the expected value of $0.36 \mathrm{pc}$ from the equipartition jet model. The structure in the sixth epoch is very similar to the fifth epoch but with lower flux density, which again is expected since the turnover frequency stayed around $10 \mathrm{GHz}$.

The separation of the outer components for all six epochs is plotted in Fig. 13 (upper panel). For the first three epochs we measure an upper limit for the expansion speed of $0.04 c$. The rapid expansion between the fourth and fifth epochs shows an apparent speed of $1.25 \mathrm{c}$. Between the last two epochs we again detected no expansion with an upper limit of $0.04 c$.

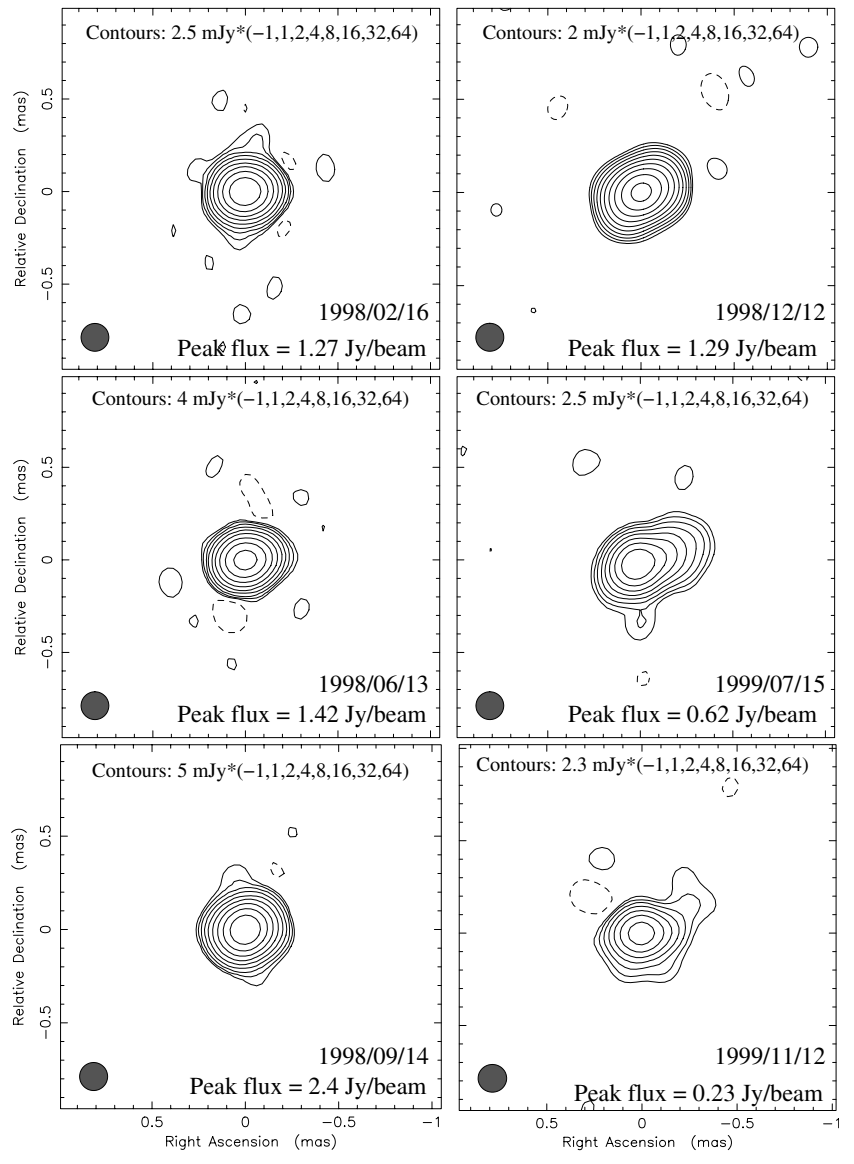

Fig. 12. All six VLBA maps of III Zw 2 at $43 \mathrm{GHz}$ convolved with a superresolved circular beam of $150 \mu$ as.

Unfortunately the source was too weak at $43 \mathrm{GHz}$ to be detected in the last three epochs.

\subsection{2. $15 \mathrm{GHz}$ results}

At $15 \mathrm{GHz}$ the picture looks completely different. The source is very compact but slightly resolved in all epochs except the first, so we fitted two point-like components to the uv-data. The flux densities, separations, and position angles of the two components are listed in Table 4. The component separation of all epochs is also plotted in Fig. 13. One can see constant expansion with apparent expansion speed of $\sim 0.6 c$. Simple extrapolation backwards suggests that the expansion started in May 1996, which is consistent with the onset of the new flare in the $37 \mathrm{GHz}$ lightcurve in Fig. 4. The flare started between two $37 \mathrm{GHz}$ observations in May and October 1996.

The component separation of the fifth epoch shows deviation from constant expansion. If one splits up the separation into its north-south and east-west components, the scatter is larger in the north-south direction. This is expected, since the beam of the VLBA is elongated in the north-south direction. This relatively large scatter in north-south direction also explains the scatter in position angle in Table 4. 


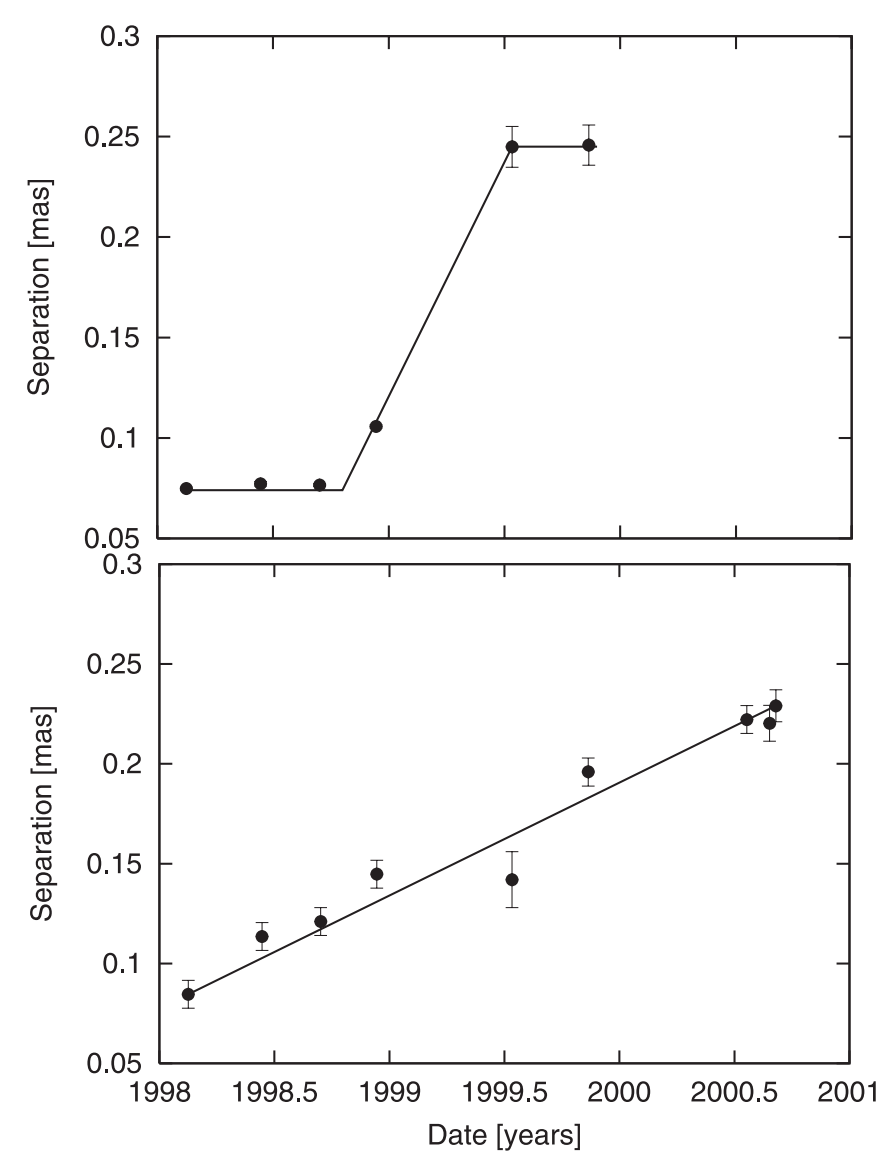

Fig. 13. Component separation at 43 (upper) and 15 (lower) GHz. The apparent expansion speed is $0.6 c$ at $15 \mathrm{GHz}$ and $1.25 c$ at $43 \mathrm{GHz}$.

\section{Discussion}

The stop-and-go behavior and the apparent contradiction between the $43 \mathrm{GHz}$ and $15 \mathrm{GHz}$ data can be explained by a jet interacting with the interstellar medium in combination with optical depth effects in an "inflating-balloon model".

In this model, the initial phase of the flare can be explained by a relativistic jet interacting with the interstellar medium or a torus that creates a shock and gets frustrated. A relativistic shock was proposed by Falcke et al. (1999) due to synchrotron cooling times of 14-50 days, which are much shorter than the duration of the outburst. The ultra-compact hotspots are pumped up, powered by the jet, and therefore responsible for the increase in flux density. The post-shock material expands with the maximum sound speed of a magnetized relativistic plasma of $c_{\mathrm{s}} \approx 0.6 c$.

Since the source is optically thick at $15 \mathrm{GHz}$, one observes the outside of the source, i.e. the post-shock material expanding with sound speed. At $43 \mathrm{GHz}$, the source is optically thin so one can look inside it to see the stationary hotspots. Rapid expansion at $43 \mathrm{GHz}$ thereafter characterised the phase where the jet breaks free and starts to propagate relativistically into a lower-density medium. Then the expansion stops again when the jet hits another cloud.

The close link between spectral and structural evolution during the outburst demonstrates that we are dealing with a true physical expansion and not simply with a phase velocity.
Table 4. Flux densities $S$, separation $D$, and position angle PA of the two point-like components of our model-fits to the $15 \mathrm{GHz}$ uv-data.

\begin{tabular}{lclcl}
\hline \hline Date & $S_{1}[\mathrm{Jy}]$ & $S_{2}[\mathrm{Jy}]$ & $D[\mathrm{mas}]$ & $\mathrm{PA}$ \\
\hline $1998 / 02 / 16$ & 0.32 & 0.40 & 0.085 & $-54^{\circ}$ \\
$1998 / 06 / 13$ & 0.45 & 0.48 & 0.114 & $-79^{\circ}$ \\
$1998 / 09 / 14$ & 0.67 & 0.50 & 0.121 & $-70^{\circ}$ \\
$1998 / 12 / 12$ & 0.79 & 0.69 & 0.145 & $-59^{\circ}$ \\
$1999 / 07 / 15$ & 0.50 & 0.46 & 0.142 & $-87^{\circ}$ \\
$1999 / 11 / 12$ & 0.43 & 0.25 & 0.195 & $-68^{\circ}$ \\
$2000 / 07 / 22$ & 0.22 & 0.10 & 0.222 & $-61^{\circ}$ \\
$2000 / 08 / 27$ & 0.19 & 0.08 & 0.220 & $-68^{\circ}$ \\
$2000 / 09 / 06$ & 0.18 & 0.07 & 0.229 & $-63^{\circ}$ \\
\hline
\end{tabular}

The observations described here have produced a large amount of data that are all consistent with a simple synchrotron selfabsorbed jet model.

In relation to the question of the nature of the radioloud/radio-quiet dichotomy our results mean that radio-weak and radio-loud quasars can indeed be powered by central engines that are very similar in many respects. Their optical properties are almost indistinguishable, and both types of quasars can produce relativistic jets in their nuclei. Discovery of superluminal motion supports the hypothesis of Miller et al. (1993) and of Falcke et al. (1996a) that RIQs are relativistically boosted intrinsically radio-weak AGN. Recently, a further relativistic jet in a radio-quiet quasar was found by Blundell et al. (2003).

However, the nature of the medium interacting with the jet remains unclear. The outbursts could be explained by a precessing jet that hits a molecular torus roughly every five years. So far, no direct evidence for molecular gas in the nucleus of III Zw 2 was found.

Some Seyfert galaxies have shown $\mathrm{H}_{2} \mathrm{O}$ maser emission associated with the nuclear jet. In these sources, the maser emission results from interaction of the jet with a molecular cloud. One example is the Seyfert II galaxy Mrk 348 (see Peck et al. 2003). In this source, ejection of a new VLBI component led to a flare of the radio source similar to the outburst in III Zw 2. The outburst started with a peak frequency of $\approx 22 \mathrm{GHz}$, which gradually decreased over 20 months. During this outburst, $\mathrm{H}_{2} \mathrm{O}$ maser emission was found (Falcke et al. 2000). Several attempts to find similar water maser emission in III Zw 2 with the Effelsberg 100-m telescope yielded nothing (Henkel, private communication).

In the currently favoured youth model for Compact Steep Spectrum (CSS) and GHz Peaked Spectrum (GPS) sources, the linear size of a source is related to its age. The correlation between turnover frequency and the projected linear size (e.g., O'Dea \& Baum 1997) suggests that the turnover frequency decreases while the source ages and expands. Therefore the sources with the highest turnover frequencies represent the youngest objects. In Fig. 14 we plot linear size vs. turnover frequency for GPS and CSS sources. We include III Zw 2 before $\left(v_{\mathrm{ssa}} \approx 33 \mathrm{GHz}\right.$; size $\left.\approx 0.11 \mathrm{pc}\right)$ and $\operatorname{after}\left(v_{\mathrm{ssa}} \approx 10 \mathrm{GHz}\right.$; size $\approx 0.37 \mathrm{pc})$ the expansion, and the two points lie at the lower end of the scatter of the linear correlation for GPS/CSS sources. 


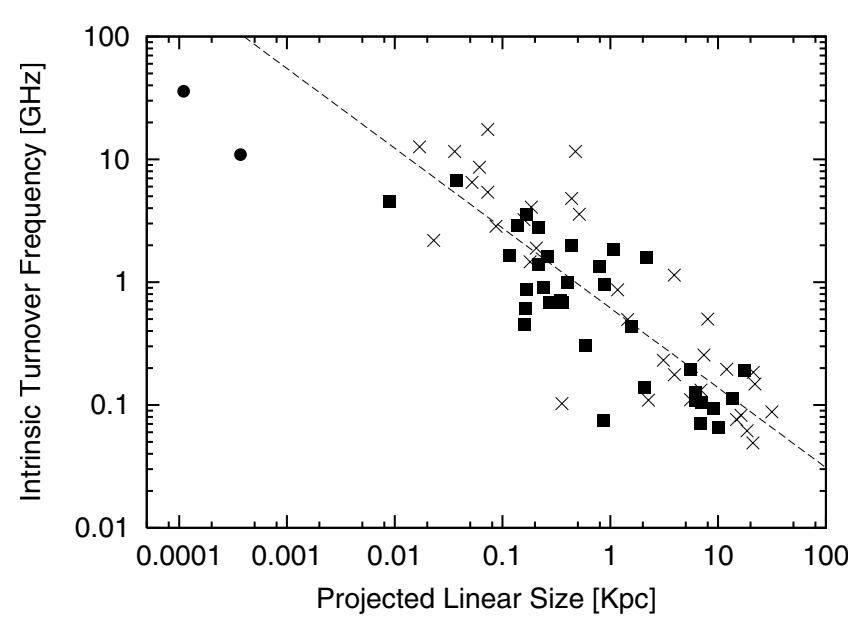

Fig. 14. Intrinsic turnover frequency vs. linear size for GPS and CSS sources. The quasars are represented by crosses, and the galaxies by solid squares. Adapted from O'Dea \& Baum (1997). The two circles mark values for III Zw 2 before and after the expansion at $43 \mathrm{GHz}$.

This could be explained by projection effects. Since III Zw 2 is a Seyfert 1 galaxy with superluminal motion, the jet is probably close to the line-of-sight, leading to underestimation of the true size and a shift of the points in the plot to the left. However, that the evolution of III Zw 2 during the expansion is almost parallel to the correlation, implies that the same physical processes, i.e. synchrotron self-absorption, are involved in III Zw 2 and in GPS/CSS sources. In the case of III Zw 2, the radio source is much older than the current outburst. So, it is possible that some of the GPS/CSS sources are in fact not young, but only show intermittent activity.

III Zw 2 remains an extremely unusual object. Future simultaneous multi-frequency observations of new outbursts would help to confirm the proposed scenario of a jet-ISM interaction.

Acknowledgements. The National Radio Astronomy Observatory is a facility of the National Science Foundation operated under cooperative agreement by Associated Universities, Inc. The UMRAO is partially supported by funds from the National Science Foundation and from the Univ. of Michigan Dept. of Astronomy. The $100 \mathrm{~m}$ telescope at Effelsberg is operated by the Max-Planck-Institut für Radioastronomie in Bonn.

\section{References}

Aller, H. D., Aller, M. F., Latimer, G. E., \& Hodge, P. E. 1985, ApJS, 59,513
Alonso-Herrero, A., Ward, M. J., \& Kotilainen, J. K. 1997, MNRAS, 288, 977

Arp, H. 1968, ApJ, 152, 1101

Bahcall, J. N., Kirhakos, S., \& Schneider, D. P. 1995, ApJ, 450, 486

Blandford, R. D., \& Konigl, A. 1979, ApJ, 232, 34

Blundell, K. M., \& Beasley, A. J. 1998, MNRAS, 299, 165

Blundell, K. M., Beasley, A. J., \& Bicknell, G. V. 2003, ApJ, 591, L103

Boroson, T. A., \& Green, R. F. 1992, ApJS, 80, 109

Brunthaler, A., Falcke, H., Bower, G. C., et al. 2000, A\&A, 357, L45

Clements, S. D., Smith, A. G., Aller, H. D., \& Aller, M. F. 1995, AJ, 110,529

Falcke, H., \& Biermann, P. L. 1995, A\&A, 293, 665

Falcke, H., Patnaik, A. R., \& Sherwood, W. 1996a, ApJ, 473, L13

Falcke, H., Sherwood, W., \& Patnaik, A. R. 1996b, ApJ, 471, 106

Falcke, H., Bower, G. C., Lobanov, A. P., et al. 1999, ApJ, 514, L17

Falcke, H., Henkel, C., Peck, A. B., et al. 2000, A\&A, 358, L17

Hutchings, J. B. 1983, PASP, 95, 799

Hutchings, J. B., \& Campbell, B. 1983, Nature, 303, 584

Kaastra, J. S., \& de Korte, P. A. J. 1988, A\&A, 198, 16

Kellermann, K. I., Sramek, R., Schmidt, M., Shaffer, D. B., \& Green, R. 1989, AJ, 98, 1195

Kellermann, K. I., Vermeulen, R. C., Zensus, J. A., \& Cohen, M. H. 1998, AJ, 115, 1295

Khachikian, E. Y., \& Weedman, D. W. 1974, ApJ, 192, 581

Kirhakos, S., Bahcall, J. N., Schneider, D. P., \& Kristian, J. 1999, ApJ, 520,67

Kukula, M. J., Dunlop, J. S., Hughes, D. H., \& Rawlings, S. 1998, MNRAS, 297, 366

Lloyd, C. 1984, MNRAS, 209, 697

Miller, P., Rawlings, S., \& Saunders, R. 1993, MNRAS, 263, 425

O’Dea, C. P., \& Baum, S. A. 1997, AJ, 113, 148

Osterbrock, D. E. 1977, ApJ, 215, 733

Peck, A. B., Henkel, C., Ulvestad, J. S., et al. 2003, ApJ, 590, 149

Ryle, M. S., \& Longair, M. S. 1967, MNRAS, 136, 123

Salvi, N. J., Page, M. J., Stevens, J. A., et al. 2002, MNRAS, 335, 177

Scarpa, R., Urry, C. M., Falomo, R., Pesce, J. E., \& Treves, A. 2000, ApJ, 532, 740

Schmidt, M., \& Green, R. F. 1983, ApJ, 269, 352

Shepherd, M. C., Pearson, T. J., \& Taylor, G. 1994, BAAS, 26, 987

Surace, J. A., Sanders, D. B., \& Evans, A. S. 2001, AJ, 122, 2791

Türler, M., Courvoisier, T. J.-L., \& Paltani, S. 1999, A\&A, 349, 45

Taylor, G. L., Dunlop, J. S., Hughes, D. H., \& Robson, E. I. 1996, MNRAS, 283, 930

Unger, S. W., Lawrence, A., Wilson, A. S., Elvis, M., \& Wright, A. E. 1987, MNRAS, 228, 521

Valtaoja, E., Lähteenmäki, A., Teräsranta, H., \& Lainela, M. 1999, ApJS, 120, 95

Zwicky, F. 1967, Adv. Astron. Astrophys., 5, 267 